\title{
The Approximation of Bivariate Blending Variant Szász Operators Based Brenke Type Polynomials
}

\author{
Behar Baxhaku, Ramadan Zejnullahu, and Artan Berisha \\ Department of Mathematics, University of Prishtina, Mother Teresa, 10000 Prishtina, Kosovo \\ Correspondence should be addressed to Artan Berisha; artan.berisha@uni-pr.edu
}

Received 13 July 2018; Accepted 3 October 2018; Published 2 December 2018

Academic Editor: Ivan Giorgio

Copyright (C) 2018 Behar Baxhaku et al. This is an open access article distributed under the Creative Commons Attribution License, which permits unrestricted use, distribution, and reproduction in any medium, provided the original work is properly cited.

\begin{abstract}
We have constructed a new sequence of positive linear operators with two variables by using Szasz-Kantorovich-Chlodowsky operators and Brenke polynomials. We give some inequalities for the operators by means of partial and full modulus of continuity and obtain a Lipschitz type theorem. Furthermore, we study the convergence of Szasz-Kantorovich-Chlodowsky-Brenke operators in weighted space of function with two variables and estimate the rate of approximation in terms of the weighted modulus of continuity.
\end{abstract}

\section{Introduction}

The well-known Mirakjan-Favard-Szasz type operators of one variable are defined as

$$
S_{n}(f ; x)=\sum_{i=0}^{\infty} \omega_{n}^{i} f\left(\frac{i}{n}\right), \quad n \in N, x \in[0, \infty),
$$

where

$$
\omega_{n}^{i}(x)=e^{-n x} \frac{(n x)^{i}}{i !}
$$

and $f:[0, \infty) \longrightarrow \mathbb{R}$ is such that the above exist series. For the convergence of $S_{n}(f ; x)$ to $f(x)$, usually $f$ is supposed to be the exponential growth, that is, $|f(x)| \leq \alpha e^{\beta x}$, for all $x \in[0, \infty)$, with $\alpha, \beta>0$ (see [1]). Later, in 1969, Jakimovski and Leviatan [2] investigated approximation properties of the generalization of Szász operators by means of the Appell polynomials $p_{k}(x)=\sum_{i=0}^{k} a_{i}\left(x^{k-i} /(k-i) !\right)$ which satisfy the identity

$$
g(t) e^{t x}=\sum_{k=0}^{\infty} p_{k}(x) t^{k}
$$

where $g(z)=\sum_{k=0}^{\infty} a_{k} z^{k},|z|<R,(R>1)$ and $g(1) \neq 0$. Varma and Tasdelen [3] constructed positive linear operators based on orthogonal polynomials, e.g., Brenke polynomials. Suppose that

$$
\begin{aligned}
& \mathscr{K}(t)=\sum_{k=0}^{\infty} a_{k} z^{k}, \quad \mathscr{K}(0) \neq 0, \\
& \mathscr{L}(t)=\sum_{k=0}^{\infty} b_{k} z^{k}, \quad \mathscr{L}(0) \neq 0
\end{aligned}
$$

is analytic functions in the disk $|z|<R,(R>1)$, where $a_{k}$ and $b_{k}$ are real. The generating function for these polynomials is given by

$$
\mathscr{K}(t) \mathscr{L}(t x)=\sum_{k=0}^{\infty} p_{k}(x) t^{k}
$$

from which the explicit form of $p_{k}(x)$ is as follows:

$$
p_{k}(x)=\sum_{i=0}^{k} a_{l-k} b_{l} x^{l}, \quad k=0,1,2, \ldots
$$

We suppose that

(1) $\mathscr{K}(1) \neq 0, a_{k-l} b_{l} / \mathscr{K}(1) \geq 1, k=0,1,2, \ldots$,

(2) $\mathscr{L}:[0, \infty) \longrightarrow(0, \infty)$,

(3) (5) and (6) converge for $|t|<R,(R>1)$,

(4) $\lim _{u \rightarrow \infty}\left(\mathscr{L}^{(k)}(u) / \mathscr{L}(u)\right)=1$, for $k \in\{1,2,3,4\}$. 
Atakut and Buyukyazici in [4] introduced the KantorovichSzász variant based on Brenke type polynomials defined as

$$
\begin{aligned}
S_{m} & (f ; x) \\
& =\frac{c_{m}}{\mathscr{K}(1) \mathscr{L}\left(b_{m} y\right)} \sum_{j=0}^{\infty} p_{j}\left(b_{m} y\right) \int_{j / c_{m}}^{(j+1) / c_{m}} f(t) d t,
\end{aligned}
$$

where $\left(b_{m}\right),\left(c_{m}\right)$ are strictly increasing sequences of positive numbers such that $\lim _{n \rightarrow \infty}\left(1 / c_{m}\right)=0, b_{m} / c_{m}=1+O\left(1 / c_{m}\right)$. The classical Bernstein-Chlodowsky polynomials are defined by

$$
B_{n}(f ; x)=\sum_{k=0}^{n} p_{n, k}\left(\frac{x}{a_{n}}\right) f\left(\frac{k}{n} a_{n}\right)
$$

where $p_{n, k}\left(x / a_{n}\right)=\left(\begin{array}{c}n \\ k\end{array}\right)\left(x / a_{n}\right)^{k}\left(1-x / a_{n}\right)^{n-k}, 0 \leq x \leq a_{n}$ and $\left(a_{n}\right)$ is a sequence of positive numbers with $\lim _{n \rightarrow \infty} a_{n}=\infty$ and $\lim _{n \rightarrow \infty}\left(a_{n} / n\right)=0$. In the last few decades the convergence estimation for linear positive operators is an active area of research amongst researchers. Several new operators have been introduced and their convergence behavior has been discussed (see [5-8]). In [9, 10] authors introduced a bivariate blending variant of the Szász type operators and studied local approximation properties for these operators. Also, they estimated the approximation order in terms of Peetre's K-functional and partial moduli of continuity.

In the present paper, we define new bivariate operators associated with a combination of Szasz-KantorovichChlodowsky operators based on Brenke polynomials as follows:

$$
\begin{gathered}
T_{n, m, a_{n}}^{b_{m}, c_{m}}(f ; x, y)=\frac{n c_{m}}{a_{n} \mathscr{K}(1) \mathscr{L}\left(b_{m} y\right)} \sum_{k=0}^{n} \sum_{j=0}^{\infty} p_{n, k}\left(\frac{x}{a_{n}}\right) \\
\cdot p_{j}\left(b_{m} y\right) \int_{j / c_{m}}^{(j+1) / c_{m}} \int_{(k / n) a_{n}}^{((k+1) / n) a_{n}} f(t, s) d t d s,
\end{gathered}
$$

where the sequences $\left(a_{n}\right),\left(b_{m}\right),\left(c_{m}\right)$ are defined as above and satisfy the following conditions:

$$
\begin{aligned}
\lim _{n \longrightarrow \infty}\left(\frac{a_{n}}{n}\right) & =0, \\
\lim _{m \longrightarrow \infty}\left(\frac{1}{c_{m}}\right) & =0, \\
\frac{b_{m}}{c_{m}} & =1+O\left(\frac{1}{c_{m}}\right) .
\end{aligned}
$$

For operators defined in (36) we have

$$
\begin{aligned}
& T_{n, m, a_{n}}^{b_{m}, c_{m}}(f ; x, y) \\
& \quad={ }_{x} C_{n}^{*}\left({ }_{y}^{*} S_{m}^{b_{m}, c_{m}}(f ; x, y)\right)={ }_{y}^{*} S_{m}^{b_{m}, c_{m}}\left({ }_{x} C_{n}^{*}(f ; x, y)\right)
\end{aligned}
$$

where

$$
{ }_{x} C_{n}^{*}(f ; x, y)=\frac{n}{a_{n}} \sum_{k=0}^{n} p_{n, k}\left(\frac{x}{a_{n}}\right) \int_{(k / n) a_{n}}^{((k+1) / n) a_{n}} f(t, y) d t
$$

and

$$
\begin{aligned}
& { }_{y}^{*} S_{m}^{b_{m}, c_{m}}(f ; x, y) \\
& =\frac{b_{m}}{\mathscr{K}(1) \mathscr{L}\left(b_{m} y\right)} \sum_{j=0}^{\infty} p_{j}\left(b_{m} y\right) \int_{j / c_{m}}^{(j+1) / c_{m}} f(x, s) d s .
\end{aligned}
$$

In this study, we give some basic convergence properties for the operators defined by (9) and study local approximation properties for these operators. Furthermore, we study the linear positive operators in a weighted space of function with two variables and estimate the rate of approximation of the operators $T_{n, m, a_{n}}^{b_{m}, c_{m}}$ in the terms of the weighted modulus of continuity.

\section{Notations and Auxiliary Results}

We will subsequently need the following basic results to prove the main results.

In what follows, let $e_{i j}(x, y)=x^{i} y^{j},(i, j) \in \mathbb{N}^{0} \times \mathbb{N}^{0}$, where $i+j \leq 4$ is the two dimensional test functions.

By simple calculations we get the following lemma.

Lemma 1. Let $T_{n, m, a_{n}}^{b_{m}, c_{m}}$ be the bivariate of Szasz-KantorovichChlodowsky-Brenke operators defined by (9). For all $m, n \in N$, $T_{n, m, a_{n}}^{b_{m}, c_{m}}$ satisfy the following results:

(i)

$$
T_{n, m, a_{n}}^{b_{m}, c_{m}}\left(e_{00} ; x, y\right)=1
$$

(ii)

$$
T_{n, m, a_{n}}^{b_{m}, c_{m}}\left(e_{10} ; x, y\right)=x+\frac{a_{n}}{2 n}
$$

(iii)

$$
T_{n, m, a_{n}}^{b_{m}, c_{m}}\left(e_{01} ; x, y\right)=\frac{b_{m} y \mathscr{L}^{\prime}\left(b_{m} y\right)}{c_{m} \mathscr{L}\left(b_{m} y\right)}+\frac{2 \mathscr{K}^{\prime}(1)+1}{2 \mathscr{K}(1) c_{m}} ;
$$

(iv)

$$
T_{n, m, a_{n}}^{b_{m}, c_{m}}\left(e_{20} ; x, y\right)=\left(1-\frac{1}{n}\right) x^{2}+2 \frac{a_{n}}{n} x+\frac{a_{n}^{2}}{3 n^{2}} ;
$$

$(v)$

$$
\begin{aligned}
T_{n, m, a_{n}}^{b_{m}, c_{m}}\left(e_{02} ; x, y\right) & \\
= & \frac{b_{m}^{2} \mathscr{L}^{\prime \prime}\left(b_{m} y\right) y^{2}}{c_{m}^{2} \mathscr{L}\left(b_{m} y\right)} \\
& +\frac{2 b_{m}\left(\mathscr{K}^{\prime}(1)+\mathscr{K}(1)\right) \mathscr{L}^{\prime}\left(b_{m} y\right) y}{c_{m}^{2} \mathscr{K}(1) \mathscr{L}\left(b_{m} y\right)} \\
& +\frac{3 \mathscr{K}^{\prime \prime}(1)+6 \mathscr{K}^{\prime}(1)+\mathscr{K}(1)}{3 c_{m}^{2} \mathscr{K}(1)}
\end{aligned}
$$


(vi)

$$
\begin{aligned}
& T_{n, m, a_{n}}^{b_{m}, c_{m}}\left(e_{03} ; x, y\right) \\
& =\frac{b_{m}^{3} \mathscr{L}^{\prime \prime \prime}\left(b_{m} y\right) y^{3}}{c_{m}^{3} \mathscr{L}\left(b_{m} y\right)} \\
& \quad+\frac{3 b_{m}^{2}\left(2 \mathscr{K}^{\prime}(1)+3 \mathscr{K}(1)\right) \mathscr{L}^{\prime \prime}\left(b_{m} y\right) y^{2}}{c_{m}^{3} \mathscr{K}(1) \mathscr{L}\left(b_{m} y\right)}
\end{aligned}
$$

$$
\begin{aligned}
& +\frac{b_{m}\left(6 \mathscr{K}^{\prime \prime}(1)+18 \mathscr{K}^{\prime}(1)+7 \mathscr{K}(1)\right) \mathscr{L}^{\prime}\left(b_{m} y\right) y}{2 c_{m}^{3} \mathscr{K}(1) \mathscr{L}\left(b_{m} y\right)} \\
& +\frac{4 \mathscr{K}^{\prime \prime \prime}(1)+16 \mathscr{K}^{\prime}(1)+12 \mathscr{K}^{\prime}(1)+7 \mathscr{K}(1)}{4 c_{m}^{3} \mathscr{K}(1)}
\end{aligned}
$$

$$
\begin{aligned}
T_{n, m, a_{n}}^{b_{m}, c_{m}}\left(e_{04} ; x, y\right)= & \frac{b_{m}^{4} \mathscr{L}^{(4)}\left(b_{m} y\right) y^{4}}{c_{m}^{4}}+\frac{4 b_{m}^{3}\left(\mathscr{K}^{\prime}(1)+2 \mathscr{K}(1)\right) \mathscr{L}^{\prime \prime \prime}\left(b_{m} y\right) y^{3}}{c_{m}^{4} \mathscr{K}(1) \mathscr{L}\left(b_{m} y\right)} \\
& +\frac{3 b_{m}^{2}\left(2 \mathscr{K}^{\prime \prime}(1)+8 \mathscr{K}^{\prime}(1)+5 \mathscr{K}(1)\right) \mathscr{L}^{\prime \prime}\left(b_{m} y\right) y^{2}}{2 c_{m}^{4} \mathscr{K}(1) \mathscr{L}\left(b_{m} y\right)} \\
& +\frac{b_{m}\left(4 \mathscr{K}^{\prime \prime \prime}(1)+24 \mathscr{K}^{\prime \prime}(1)+30 \mathscr{K}^{\prime}(1)+6 \mathscr{K}(1)\right) \mathscr{L}^{\prime}\left(b_{m} y\right) y}{c_{m}^{4} \mathscr{K}(1) \mathscr{L}\left(b_{m} y\right)} \\
& +\frac{5 \mathscr{K}^{(4)}(1)+40 \mathscr{K}^{\prime \prime \prime}(1)+75 \mathscr{K}^{\prime \prime}(1)+30 \mathscr{K}^{\prime}(1)+\mathscr{K}(1)}{c_{m}^{4} \mathscr{K}(1)} .
\end{aligned}
$$

Proof. In view of definition of operators defined by (9) we have

$$
\begin{aligned}
& T_{n, m, a_{n}}^{b_{m}, c_{m}}\left(e_{00} ; x, y\right)={ }_{x} C_{n}^{*}\left(e_{0} ; x, y\right)_{y}^{*} S_{m}^{b_{m}, c_{m}}\left(e_{0} ; x, y\right) \\
& T_{n, m, a_{n}}^{b_{m}, c_{m}}\left(e_{10} ; x, y\right)={ }_{x} C_{n}^{*}\left(e_{1} ; x, y\right)_{y}^{*} S_{m}^{b_{m}, c_{m}}\left(e_{0} ; x, y\right) \\
& T_{n, m, a_{n}}^{b_{m}, c_{m}}\left(e_{01} ; x, y\right)={ }_{x} C_{n}^{*}\left(e_{0} ; x, y\right)_{y}^{*} S_{m}^{b_{m}, c_{m}}\left(e_{1} ; x, y\right) \\
& T_{n, m, a_{n}}^{b_{m}, c_{m}}\left(e_{20} ; x, y\right)={ }_{x} C_{n}^{*}\left(e_{2} ; x, y\right)_{y}^{*} S_{m}^{b_{m}, c_{m}}\left(e_{0} ; x, y\right) \\
& T_{n, m, a_{n}}^{b_{m}, c_{m}}\left(e_{02} ; x, y\right)={ }_{x} C_{n}^{*}\left(e_{0} ; x, y\right)_{y}^{*} S_{m}^{b_{m}, c_{m}}\left(e_{2} ; x, y\right) \\
& T_{n, m, a_{n}}^{b_{m}, c_{m}}\left(e_{03} ; x, y\right)={ }_{x} C_{n}^{*}\left(e_{0} ; x, y\right)_{y}^{*} S_{m}^{b_{m}, c_{m}}\left(e_{3} ; x, y\right) \\
& T_{n, m, a_{n}}^{b_{m}, c_{m}}\left(e_{04} ; x, y\right)={ }_{x} C_{n}^{*}\left(e_{0} ; x, y\right)_{y}^{*} S_{m}^{b_{m}, c_{m}}\left(e_{4} ; x, y\right)
\end{aligned}
$$

with the help of these equalities, we can easily prove required results.

Lemma 2. It follows from Lemma 1 that

$$
\begin{aligned}
& T_{n, m, a_{n}}^{b_{m}, c_{m}}\left(e_{10}-x ; x, y\right)=\frac{a_{n}}{2 n} ; \\
& T_{n, m, a_{n}}^{b_{m}, c_{m}}\left(e_{10}-x ; x, y\right)=\left(\frac{b_{m}^{2} \mathscr{L}^{\prime \prime}\left(b_{m} y\right)}{c_{m}^{2} \mathscr{L}\left(b_{m} y\right)}-1\right) y \\
& \quad+\frac{2 \mathscr{K}^{\prime}(1)+1}{2 c_{m} \mathscr{K}(1)} ; \\
& T_{n, m, a_{n}}^{b_{m}, c_{m}}\left(\left(e_{10}-x\right)^{2} ; x, y\right)=\frac{x\left(a_{n}-x\right)}{n}+\frac{a_{n}^{2}}{3 n^{2}}
\end{aligned}
$$

$$
\begin{aligned}
& T_{n, m, a_{n}}^{b_{m}, c_{m}}\left(\left(e_{01}-y\right)^{2} ; x, y\right)=\left(\frac{b_{m}^{2} \mathscr{L}^{\prime \prime}\left(b_{m} y\right)}{c_{m}^{2} \mathscr{L}\left(b_{m} y\right)}\right. \\
& \left.-\frac{2 b_{m} \mathscr{L}^{\prime}\left(b_{m} y\right)}{c_{m} \mathscr{L}\left(b_{m} y\right)}+1\right) y^{2} \\
& +\left(\frac{2 b_{m}\left(\mathscr{K}^{\prime}(1)+\mathscr{K}(1)\right) \mathscr{L}^{\prime}\left(b_{m} y\right)}{c_{m}^{2} \mathscr{K}(1) \mathscr{L}\left(b_{m} y\right)}\right. \\
& \left.-\frac{2 \mathscr{K}^{\prime}(1)+1}{c_{m} \mathscr{K}(1)}\right) y+\frac{3 \mathscr{K}^{\prime \prime}(1)+6 \mathscr{K}^{\prime}(1)+\mathscr{K}(1)}{3 c_{m}^{2} \mathscr{K}(1)} .
\end{aligned}
$$

Proof. The results follow from linearity of the operators $T_{n, m, a_{n}}^{b_{m}, c_{m}}$ and Lemma 1.

For sufficiently large $n, m$, for all $(x, y) \in I_{a_{n}}$, by taking into consideration Lemma 1, and condition (10), we have the following equalities:

$$
\begin{aligned}
\Delta_{1} & =T_{n, m, a_{n}}^{b_{m}, c_{m}}\left(\left(e_{10}-x\right)^{2} ; x, y\right) \\
& { }_{x} C_{n}^{*}\left(\left(e_{10}-x\right)^{2} ; x, y\right)=O\left(\frac{a_{n}}{n}\right) \sum_{i=1}^{2} x^{i} ; \\
\Delta_{2} & =T_{n, m, a_{n}}^{b_{m}, c_{m}}\left(\left(e_{10}-x\right)^{4} ; x, y\right) \\
& { }_{x} C_{n}^{*}\left(\left(e_{10}-x\right)^{4} ; x, y\right)=O\left(\frac{a_{n}}{n}\right) \sum_{i=1}^{4} x^{i} ;
\end{aligned}
$$


TABLE 1: Error estimation for operator (9) to the function $f(x, y)=x^{2} y-x y^{2}$ for $n=m=100$.

\begin{tabular}{lccccccc}
\hline $\mathrm{x}$ & $(0.01,0.5)$ & $(0.1,0.5)$ & $(0.4,0.5)$ & $(0.5,0.5)$ & $(0.6,0.5)$ & $(0.8,0.5)$ & $(0.9,0.5)$ \\
\hline $\mathrm{n}=\mathrm{m}=1000$ & 0.0037 & 0.0011 & 0.0076 & 0.0105 & 0.0135 & 0.0196 & 0.0226 \\
\hline $\mathrm{n}=\mathrm{m}=100$ & 0.0118 & 0.0052 & 0.0187 & 0.0273 & 0.0361 & 0.0548 & 0.0645 \\
\hline
\end{tabular}

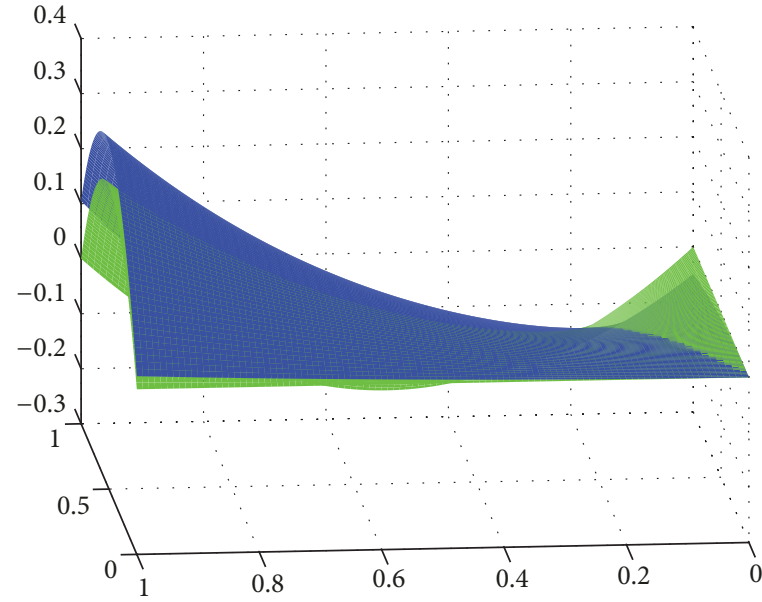

Operators Function

(a)

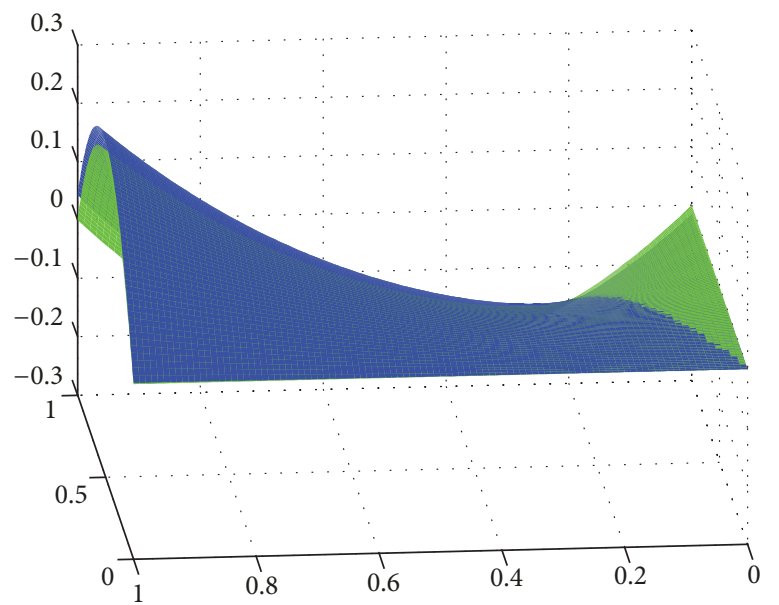

Operators Function

(b)

Figure 1

and

$$
\begin{aligned}
\Delta_{1}^{*} & =T_{n, m, a_{n}}^{b_{m}, c_{m}}\left(\left(e_{10}-y\right)^{2} ; x, y\right) \\
& ={ }_{y}^{*} S_{m}^{b_{m}, c_{m}}\left(\left(e_{10}-y\right)^{2} ; x, y\right)=O\left(\frac{1}{c_{m}}\right) \sum_{i=1}^{2} y^{i} ; \\
\Delta_{2}^{*} & =T_{n, m, a_{n}}^{b_{m}, c_{m}}\left(\left(e_{10}-y\right)^{4} ; x, y\right) \\
& ={ }_{y}^{*} S_{m}^{b_{m}, c_{m}}\left(\left(e_{10}-y\right)^{4} ; x, y\right)=O\left(\frac{1}{c_{m}}\right) \sum_{i=0}^{4} y^{i} .
\end{aligned}
$$

Further, let $\delta_{n}(x)=\Delta_{1}, \delta_{m}(y)=\Delta_{1}^{*}$, and $\delta_{m}(x, y)=\left(\Delta_{1}+\right.$ $\left.\Delta_{1}^{*}\right)^{1 / 2}$.

\section{Main Results}

To study the convergence of the sequence $\left\{T_{n, m, a_{n}}^{b_{m}, c_{m}}\right\}$ we shall use the following Korovkin type theorem, established by Volkov [11]. Next, the degree of approximation of the operator $\left\{T_{n, m, a_{n}}^{b_{m}, c_{m}}\right\}$ given by (36) will be established in the space of continuous function on compact set $I_{a b}=[0, a] \times[0, b] \subset I_{a_{n}}$. For $I_{a b}=[0, a] \times[0, b]$, let $C\left(I_{a b}\right)$, denote the space of all real valued continuous functions on $I_{a b}$, endowed with the norm $\|f\|_{C\left(I_{a b}\right)}=\sup _{(x, y) \in I_{a b}}|f(x, y)|$.

Theorem 3. Let $\left\{T_{n, m, a_{n}}^{b_{m}, c_{m}}\right\}$ be the sequences of linear positive operators defined by (36). Then for each $f \in C\left(I_{a b}\right)$, we have $\lim _{a, m \rightarrow \infty} T_{n, m, a_{n}}^{b_{m}, c_{m}}=f(x, y)$ uniformly on the compact set

Proof. From Lemma 1, we have

$$
\begin{aligned}
\lim _{n, m \rightarrow \infty} T_{n, m, a_{n}}^{b_{m}, c_{m}}\left(e_{i j} ; x, y\right)= & e_{i j}=0, \\
& (i, j) \in\{(0,0),(1,1),(2,2)\}
\end{aligned}
$$

and

$$
\lim _{n, m \longrightarrow \infty} T_{n, m, a_{n}}^{b_{m}, c_{m}}\left(e_{20}+e_{02} ; x, y\right)=e_{20}+e_{02}
$$

uniformly on $I_{a b}$. The result follows from the well-known Volkov theorem.

Example 4. Let us consider the function $f(x, y)=x^{2} y-x y^{2}$. For $n=m=100,1000, \mathscr{K}(t)=e^{2 t} ; \mathscr{L}(t)=e^{t}$ and $a_{n}=\sqrt{n}$; $b_{m}=m ; c_{m}=m+1 / \sqrt{m}$ the convergence of $T_{n, m, a_{n}}^{b_{m}, c_{m}}$ to $f(x, y)$ is illustrated in Figures 1(a) and 1(b), respectively. Further, in Table 1 we compute error estimation for operator (9) to the function $f$.

Example 5. For $\mathscr{K}(t)=e^{2 t}, \mathscr{L}(t)=e^{t}$ the convergence of operators $T_{n, m, a_{n}}^{b_{m}, c_{m}}$ to function $f(x, y)$ is illustrated in Figures 2(a) and 2(b), respectively, where $(x, y)=x y+x y^{2}, n=m=$ 100,1000 , and $a_{n}=\sqrt{n} ; b_{m}=m ; c_{m}=m+1 / \sqrt{m}$. In Table 2 there are are compute error estimations for operator (9) to the function $f$. 
TABLE 2: Error estimation for operator (9) to the function $f(x, y)=x y+x y^{2}$ for $n=m=1000$.

\begin{tabular}{lccccccc}
\hline $\mathrm{x}$ & $(0.01,0.5)$ & $(0.1,0.5)$ & $(0.3,0.5)$ & $(0.4,0.5)$ & $(0.6,0.5)$ & $(0.8,0.5)$ & $(0.9,0.5)$ \\
\hline $\mathrm{n}=\mathrm{m}=1000$ & 0.0116 & 0.0121 & 0.0131 & 0.0136 & 0.0151 & 0.0156 & 0.0161 \\
\hline $\mathrm{n}=\mathrm{m}=100$ & 0.0390 & 0.0435 & 0.0535 & 0.0584 & 0.0734 & 0.0784 & 0.0834 \\
\hline
\end{tabular}

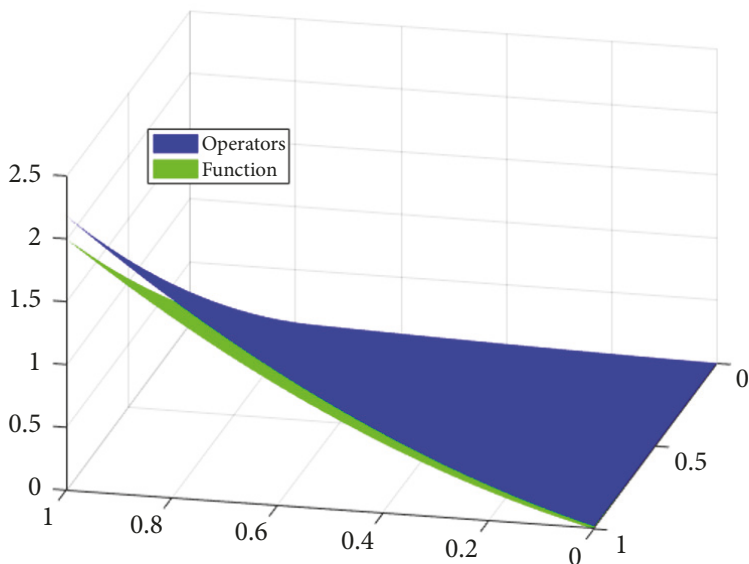

(a)

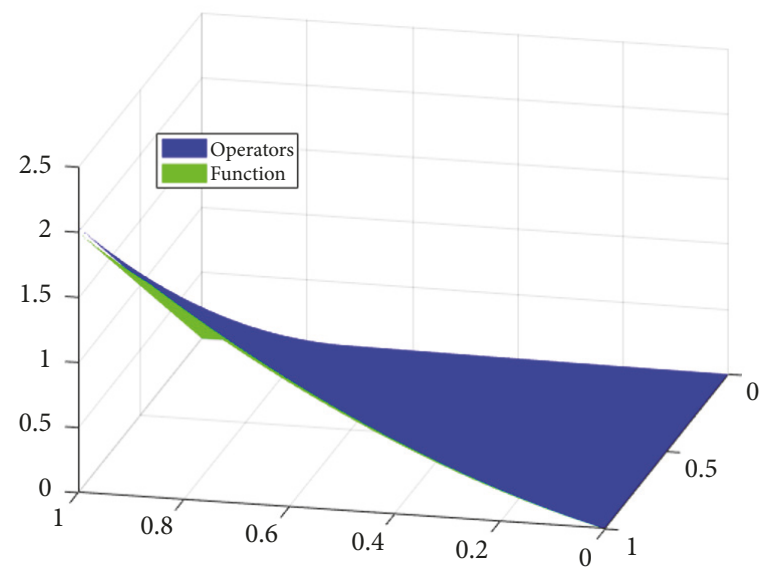

(b)

Figure 2

An estimation of the rate of convergence can be obtained using the modulus of continuity for two dimensional real valued functions. Let $f \in C\left(I_{a b}\right)$ and $\delta>0$. In what follows, we shall use the following modulus of continuity for bivariate real functions:

$$
\begin{aligned}
& \omega\left(f ; \delta_{n}, \delta_{m}\right)=\sup \{|f(t, s)-f(x, y)|:(t, s),(x, y) \\
& \left.\quad \in I_{a b},|t-x| \leq \delta_{n},|s-y| \leq \delta_{m}\right\} .
\end{aligned}
$$

Alternately, the complete modulus of continuity of $f$ which we denote by $\omega(f ; \delta)$ is defined as

$$
\begin{aligned}
& \omega(f ; \delta) \\
& \quad=\frac{\sup }{\sqrt{(t-x)^{2}+(s-y)^{2}} \leq \delta}\{|f(t, s)-f(x, y)|:(t, s),(x, y) \\
& \left.\quad \in I_{a b},\right\} .
\end{aligned}
$$

Theorem 6. For any $f \in C\left(I_{a b}\right)$, then we have estimated

$$
\left|T_{n, m, a_{n}}^{b_{m}, c_{m}}(f ; x, y)-f(x, y)\right| \leq 2 \omega\left(f ; \delta_{n, m}\right)
$$

where $\delta_{n, m}=\delta_{n, m}(x, y)$.
Proof. From (9) and by definition of $\omega(f ; \delta)$, we can write

$$
\begin{aligned}
& \left|T_{n, m, a_{n}}^{b_{m}, c_{m}}(f ; x, y)-f(x, y)\right| \leq \frac{n c_{m}}{a_{n} \mathscr{K}(1) \mathscr{L}\left(b_{m} y\right)} \\
& \cdot \sum_{k=0}^{n} \sum_{j=0}^{\infty} p_{n, k}\left(\frac{x}{a_{n}}\right) p_{j}\left(b_{m} y\right) \\
& \cdot \int_{j / c_{m}}^{(j+1) / c_{m}} \int_{(k / n) a_{n}}^{((k+1) / n) a_{n}}\left(\omega\left(f ; \sqrt{(t-x)^{2}+(s-y)^{2}} ; x, y\right)\right) d t d s \\
& \quad \leq \omega\left(f ; \delta_{n, m}\right)\left\{1+\frac{1}{\delta_{m, n}} \frac{n c_{m}}{a_{n} \mathscr{K}(1) \mathscr{L}\left(b_{m} y\right)}\right. \\
& \quad \cdot \sum_{k=0}^{n} \sum_{j=0}^{\infty} p_{n, k}\left(\frac{x}{a_{n}}\right) p_{j}\left(b_{m} y\right) \\
& \left.\cdot \int_{j / c_{m}}^{(j+1) / c_{m}} \int_{(k / n) a_{n}}^{((k+1) / n) a_{n}} \sqrt{(t-x)^{2}+(s-y)^{2}} d t d s\right\}
\end{aligned}
$$

Using the Cauchy-Schwarz inequality, we obtain

$$
\begin{aligned}
& \left|T_{n, m, a_{n}}^{b_{m}, c_{m}}(f ; x, y)-f(x, y)\right| \leq \omega\left(f ; \delta_{n, m}\right)(1 \\
& +\frac{1}{\delta_{n, m}}\left(T_{n, m, a_{n}}^{b_{m}, c_{m}}\left((t-x)^{2}+(s-y)^{2} ; x, y\right)\right)^{1 / 2} \\
& \quad \leq \omega\left(f ; \delta_{n, m}\right)(1 \\
& \left.\quad+\frac{1}{\delta_{n, m}}\left(O\left(\frac{a_{n}}{n}\right) \sum_{i=1}^{2} x^{i}+O\left(\frac{1}{c_{m}}\right) \sum_{i=1}^{2} y^{i}\right)^{1 / 2}\right) .
\end{aligned}
$$

Taking $\delta_{n, m}=\delta_{n, m}(x, y)$, we obtain the desired result. 
The partial modulus of continuity with respect to $x$ and $y$ is given by

$$
\begin{aligned}
& \omega^{(1)}(f ; \delta)=\sup _{0 \leq y \leq b} \sup _{\left|x_{1}-x_{2}\right| \leq \delta}\left\{\left|f\left(x_{1}, y\right)-f\left(x_{2}, y\right)\right|\right\}, \\
& \omega^{(2)}(f ; \delta)=\sup _{0 \leq x \leq a} \sup _{\left|y_{1}-y_{2}\right| \leq \delta}\left\{\left|f\left(x, y_{1}\right)-f\left(x, y_{2}\right)\right|\right\},
\end{aligned}
$$

Theorem 7. For any $f \in C\left(I_{a b}\right)$, then the inequalities satisfy

$$
\begin{aligned}
& \left|T_{n, m, a_{n}}^{b_{m}, c_{m}}(f ; x, y)-f(x, y)\right| \\
& \quad \leq 2\left(\omega^{(1)}\left(f ; \delta_{n}\right)+\omega^{(2)}\left(f ; \delta_{m}\right)\right)
\end{aligned}
$$

where $\delta_{n}=\delta_{n}(x), \delta_{m}=\delta_{m}(y)$.

Proof. Using the definition of partial modulus of continuity $\omega^{(i)}(f ; \delta), \quad i=1,2$, we may write

$$
\begin{aligned}
& \left|T_{n, m, a_{n}}^{b_{m}, c_{m}}(f ; x, y)-f(x, y)\right| \leq \frac{n c_{m}}{a_{n} \mathscr{K}(1) \mathscr{L}\left(b_{m} y\right)} \\
& \cdot \sum_{k=0}^{n} \sum_{j=0}^{\infty} p_{n, k}\left(\frac{x}{a_{n}}\right) p_{j}\left(b_{m} y\right) \\
& \cdot \int_{j / c_{m}}^{(j+1) / c_{m}} \int_{(k / n) a_{n}}^{((k+1) / n) a_{n}}|f(t, s)-f(t, y)| d t d s \\
& +\frac{n c_{m}}{a_{n} \mathscr{K}(1) \mathscr{L}\left(b_{m} y\right)} \sum_{k=0}^{n} \sum_{j=0}^{\infty} p_{n, k}\left(\frac{x}{a_{n}}\right) p_{j}\left(b_{m} y\right) \\
& \cdot \int_{j / c_{m}}^{(j+1) / c_{m}} \int_{(k / n) a_{n}}^{((k+1) / n) a_{n}}|f(t, y)-f(x, y)| \\
& \quad \leq \frac{n c_{m}}{a_{n} \mathscr{K}(1) \mathscr{L}\left(b_{m} y\right)} \sum_{k=0}^{n} \sum_{j=0}^{\infty} p_{n, k}\left(\frac{x}{a_{n}}\right) p_{j}\left(b_{m} y\right) \\
& \quad \cdot \int_{j / c_{m}}^{(j+1) / c_{m}} \int_{(k / n) a_{n}}^{((k+1) / n) a_{n}} \omega^{(2)}(f ;|s-y|) d t d s \\
& +\frac{n c_{m}}{a_{n} \mathscr{K}(1) \mathscr{L}\left(b_{m} y\right)} \sum_{k=0}^{n} \sum_{j=0}^{\infty} p_{n, k}\left(\frac{x}{a_{n}}\right) p_{j}\left(b_{m} y\right) \\
& \quad \cdot \int_{j / c_{m}}^{(j+1) / c_{m}} \int_{(k / n) a_{n}}^{((k+1) / n) a_{n}} \omega^{(1)}(f ;|t-x|) d t d s \\
& =\eta_{1}(x, y)+\eta_{2}(x, y) .
\end{aligned}
$$

Consider $\eta(x, y)$. Using Lemma 1 and the well-known properties of the modulus of continuity, we have

$$
\begin{aligned}
& \eta_{1}(x, y) \leq \omega^{(2)}\left(f ; \delta_{m}\right)\left[1+\frac{n c_{m}}{\delta_{m} a_{n} \mathscr{K}(1) \mathscr{L}\left(b_{m} y\right)}\right. \\
& \quad \cdot \sum_{k=0}^{n} \sum_{j=0}^{\infty} p_{n, k}\left(\frac{x}{a_{n}}\right) p_{j}\left(b_{m} y\right) \\
& \left.\quad \cdot \int_{j / c_{m}}^{(j+1) / c_{m}} \int_{(k / n) a_{n}}^{((k+1) / n) a_{n}}|s-y| d t d s\right]
\end{aligned}
$$

By using Cauchy-Schwarz inequality, we get

$$
\begin{aligned}
& \eta_{1}(x, y) \leq \omega^{(2)}\left(f ; \delta_{m}\right)\left(1+\frac{1}{\delta_{m}}\left\{\frac{n c_{m}}{a_{n} \mathscr{K}(1) \mathscr{L}\left(b_{m} y\right)}\right.\right. \\
& \quad \cdot \sum_{k=0}^{n} \sum_{j=0}^{\infty} p_{n, k}\left(\frac{x}{a_{n}}\right) p_{j}\left(b_{m} y\right)
\end{aligned}
$$$$
\left.\left.\times \int_{j / c_{m}}^{(j+1) / c_{m}} \int_{(k / n) a_{n}}^{((k+1) / n) a_{n}}(s-y)^{2} d t d s\right\}^{1 / 2}\right) .
$$

So, by using (25), we obtain

$$
\eta_{1}(x, y) \leq \omega^{(2)}\left(f ; \delta_{m}\right)\left(1+\frac{O\left(1 / c_{m}\right) \sum_{i=1}^{2} y^{i}}{\delta_{m}}\right) .
$$

In the same way we gain

$$
\eta_{2}(x, y) \leq \omega^{(1)}\left(f ; \delta_{n}\right)\left(1+\frac{O\left(a_{n} / n\right) \sum_{i=1}^{2} x^{i}}{\delta_{n}}\right) .
$$

Hence from (39), (40), and (32), we arrive at

$$
\begin{aligned}
& \left|T_{n, m, a_{n}}^{b_{m}, c_{m}}(f ; x, y)-f(x, y)\right| \\
& \quad \leq \omega^{(2)}\left(f ; \delta_{m}\right)\left(1+\frac{O\left(1 / c_{m}\right) \sum_{i=1}^{2} y^{i}}{\delta_{m}}\right) \\
& \quad+\omega^{(1)}\left(f ; \delta_{n}\right)\left(1+\frac{O\left(a_{n} / n\right) \sum_{i=1}^{2} x^{i}}{\delta_{n}}\right) .
\end{aligned}
$$

Finally, choosing $\delta_{n}=\Delta_{1}=\delta_{n}(x)$ and $\delta_{m}=\Delta_{1}^{*}=\delta_{m}(y)$, for all $(x, y) \in I_{a b}$, we reach the desired result.

For $0<\gamma \leq 1$, we define the Lipschitz class $\operatorname{Lip}_{L}(\gamma)$ for bivariate case as follows:

$$
\begin{aligned}
& \operatorname{Lip}_{L}(\gamma) \\
& :=\left\{f:\left|f\left(t_{1}, t_{2}\right)-f(x, y)\right| \leq L \frac{\|r-s\|^{\gamma}}{(\|r\|+x+y)^{\gamma / 2}}\right\},
\end{aligned}
$$

where $r=\left(t_{1}, t_{2}\right), s=(x, y)$ in $I_{a b}$, and $\|r-s\|=\left\{\left(t_{1}-x\right)^{2}+\right.$ $\left.\left(t_{2}-y\right)^{2}\right\}^{1 / 2}$ is the Euclidean norm.

Theorem 8. Suppose that $f \in \operatorname{Lip}_{L}(\gamma)$. Then, for every $(x, y) \in$ $I_{a b}$, we have

$$
\left|T_{n, m, a_{n}}^{b_{m}, c_{m}}(f ; x, y)-f(x, y)\right| \leq \frac{L \delta_{m}^{1 / 2}}{(x+y)^{1 / 2}}
$$

where $\delta_{m}=\delta_{m}(x, y)$. 
Proof. First, we prove theorem for case $\gamma=1$. Then, for $f \in$ $\operatorname{Lip}_{L}(\gamma)$ and for each $x, y \in I_{a b}$, using the monotonicity and linearity of operators, we may write

$$
\begin{aligned}
& \left|T_{n, m, a_{n}}^{b_{m}, c_{m}}(f ; x, y)-f(x, y)\right| \\
& \quad \leq T_{n, m, a_{n}}^{b_{m}, c_{m}}\left(\left|f\left(t_{1}, t_{2}\right)-f(x, y)\right| ; x, y\right) \\
& \quad \leq L T_{n, m, a_{n}}^{b_{m}, c_{m}}\left(\frac{\|r-s\|}{(\|r\|+x+y)^{1 / 2}} ; x, y\right) \\
& \quad \leq \frac{L}{(x+y)^{1 / 2}} T_{n, m, a_{n}}^{b_{m}, c_{m}}(\|r-s\| ; x, y),
\end{aligned}
$$

where $r=\left(t_{1}, t_{2}\right)$ and $s=(x, y)$.

Using the Cauchy- Shwarz inequality and Lemma 2, the above inequality implies that

$$
\begin{aligned}
& \left|T_{n, m, a_{n}}^{b_{m}, c_{m}}(f ; x, y)-f(x, y)\right| \\
& \quad \leq \frac{L}{(x+y)^{1 / 2}}\left\{T_{n, m, a_{n}}^{b_{m}, c_{m}}\left(\|r-s\|^{2}, x, y\right)\right\}^{1 / 2} \\
& \quad \leq \frac{L}{(x+y)^{1 / 2}} \\
& \cdot\left\{{ }_{x} C_{n}^{*}\left(\left(t_{1}-x\right)^{2}, x, y\right)+{ }_{y}^{*} S_{m}^{b_{m}, c_{m}}\left(\left(t_{2}-y\right)^{2}, x, y\right)\right\}^{1 / 2} \\
& \quad \leq \frac{L \delta_{m}^{1 / 2}(x, y)}{(x+y)^{1 / 2}} .
\end{aligned}
$$

Thus, the result holds for $\gamma=1$. Secondly, let $0<\gamma<1$. Then, for $f \in \operatorname{Lip}_{L}(\gamma)$ and for each $x, y \in I_{a b}$, we get

$$
\begin{aligned}
& \left|T_{n, m, a_{n}}^{b_{m}, c_{m}}(f ; x, y)-f(x, y)\right| \\
& \leq T_{n, m, a_{n}}^{b_{m}, c_{m}}\left(\left|f\left(t_{1}, t_{2}\right)-f(x, y)\right| ; x, y\right) \\
& \quad \leq L T_{n, m, a_{n}}^{b_{m}, c_{m}}\left(\frac{\|r-s\|^{\gamma}}{(\|r\|+x+y)^{\gamma / 2}} ; x, y\right) \\
& \leq \frac{L}{(x+y)^{\gamma / 2}} T_{n, m, a_{n}}^{b_{m}, c_{m}}\left(\|r-s\|^{\gamma} ; x, y\right) .,
\end{aligned}
$$

Now, applying Holder's inequality with $u_{1}=2 / \gamma, u_{2}=2 /(2-$ $\gamma$ ), and Lemma 2, we get

$$
\begin{aligned}
& \left|T_{n, m, a_{n}}^{b_{m}, c_{m}}(f ; x, y)-f(x, y)\right| \\
& \quad \leq \frac{L}{(x+y)^{\gamma / 2}}\left\{T_{n, m, a_{n}}^{b_{m}, c_{m}}\left(\|r-s\|^{2}, x, y\right)\right\}^{\gamma / 2} \\
& \quad \leq \frac{L}{(x+y)^{\gamma / 2}}\left\{C_{n}^{*}\left(\left(t_{1}-x\right)^{2}, x, y\right)++_{y}^{*} S_{m}^{b_{m}, c_{m}}\left(\left(t_{2}-y\right)^{2}, x, y\right)\right\}^{\gamma / 2} \\
& \left.\quad \leq \frac{L}{(x+y)^{1 / 2}}\left\{O\left(\frac{a_{n}}{n}\right) \sum_{i=1}^{2} x^{i}+O\left(\frac{1}{c_{m}}\right) \sum_{i=1}^{2} y^{i}\right)\right\}^{\gamma / 2} .
\end{aligned}
$$

which leads us to the required result.
Theorem 9. If $f(x, y)$ has continuous partial derivatives $f_{x}^{\prime}$ and $f_{y}^{\prime}$ and $\omega^{(1)}\left(f_{x}^{\prime} ; \delta\right)$ and $\omega^{(2)}\left(f_{y}^{\prime} ; \delta\right)$ denote the partial moduli of continuity of $f_{x}^{\prime}$ and $f_{y}^{\prime}$ respectively. Then we have estimate

$$
\begin{aligned}
\left|T_{n, m, a_{n}}^{b_{m}, c_{m}}(f ; x, y)-f(x, y)\right| \\
\leq \Lambda \frac{a_{n}}{2 n} \\
\quad+\Upsilon\left(\left|\frac{b_{m}^{2} \mathscr{L}^{\prime \prime}\left(b_{m} y\right)}{c_{m}^{2} \mathscr{L}\left(b_{m} y\right)}-1\right| y+\frac{2 \mathscr{K}^{\prime}(1)+1}{2 c_{m} \mathscr{K}(1)}\right) \\
\quad+\omega^{(1)}\left(f_{x}^{\prime} ; \delta_{n}\right)\left(1+\sqrt{\delta_{n}}\right) \\
\quad+\omega^{(2)}\left(f_{x}^{\prime} ; \delta_{m}\right)\left(1+\sqrt{\delta_{m}}\right),
\end{aligned}
$$

where $\Lambda, \Upsilon$ are the positive constants such that $|\partial f / \partial x| \leq$ $\Lambda,|\partial f / \partial y| \leq \Upsilon,\left(x, y \in I_{a b}\right)$.

Proof. From the mean value theorem we have

$$
\begin{aligned}
f\left(t_{1}, t_{2}\right)-f(x, y) & \\
= & f\left(t_{1}, y\right)-f(x, y)+f\left(t_{1}, t_{2}\right)-f\left(t_{1}, y\right) \\
= & \left(t_{1}-x\right) \frac{\partial f(\eta, y)}{\partial x}+\left(t_{2}-y\right) \frac{\partial f(x, \zeta)}{\partial y} \\
= & \left(t_{1}-x\right) \frac{\partial f(x, y)}{\partial x} \\
& +\left(t_{1}-x\right)\left(\frac{\partial f(\eta, y)}{\partial x}-\frac{\partial f(x, y)}{\partial x}\right) \\
& +\left(t_{2}-y\right) \frac{\partial f(x, y)}{\partial y} \\
& +\left(t_{2}-y\right)\left(\frac{\partial f(x, \zeta)}{\partial y}-\frac{\partial f(x, y)}{\partial y}\right),
\end{aligned}
$$

where $x<\eta<t_{1}$ and $y<\zeta<t_{2}$. By using the above identity, we get

$$
\begin{aligned}
& T_{n, m, a_{n}}^{b_{m}, c_{m}}(f ; x, y)-f(x, y) \\
& \quad=T_{n, m, a_{n}}^{b_{m}, c_{m}}\left(\left(t_{1}-x\right) \frac{\partial f(x, y)}{\partial x} ; x, y\right) \\
& \quad+T_{n, m, a_{n}}^{b_{m}, c_{m}}\left(\left(t_{1}-x\right)\left(\frac{\partial f(\eta, y)}{\partial x}-\frac{\partial f(x, y)}{\partial x}\right) ; x, y\right) \\
& +T_{n, m, a_{n}}^{b_{m}, c_{m}}\left(\left(t_{2}-y\right) \frac{\partial f(x, y)}{\partial y} ; x, y\right) \\
& \quad+T_{n, m, a_{n}}^{b_{m}, c_{m}}\left(\left(t_{2}-y\right)\left(\frac{\partial f(x, \zeta)}{\partial y}-\frac{\partial f(x, y)}{\partial y}\right) ; x, y\right) .
\end{aligned}
$$


Hence,

$$
\begin{aligned}
& \left|T_{n, m, a_{n}}^{b_{m}, c_{m}}(f ; x, y)-f(x, y)\right| \leq\left|\frac{\partial f(x, y)}{\partial x}\right| \\
& \cdot\left|T_{n, m, a_{n}}^{b_{m}, c_{m}}\left(\left(t_{1}-x\right) ; x, y\right)\right|+T_{n, m, a_{n}}^{b_{m}, c_{m}}\left(\left|t_{1}-x\right|\right. \\
& \left.\left|\frac{\partial f(\eta, y)}{\partial x}-\frac{\partial f(x, y)}{\partial x}\right| ; x, y\right)+\left|\frac{\partial f(x, y)}{\partial y}\right| \\
& \cdot\left|T_{n, m, a_{n}}^{b_{m}, c_{m}}\left(\left(t_{2}-y\right) ; x, y\right)\right|++T_{n, m, a_{n}}^{b_{m}, c_{m}}\left(\left|t_{2}-y\right|\right. \\
& \left.\left|\frac{\partial f(x, \zeta)}{\partial y}-\frac{\partial f(x, y)}{\partial y}\right| ; x, y\right) \\
& \leq \Lambda\left|T_{n, m, a_{n}}^{b_{m}, c_{m}}\left(\left(t_{1}-x\right) ; x, y\right)\right| \\
& +\Upsilon\left|T_{n, m, a_{n}}^{b_{m}, c_{m}}\left(\left(t_{2}-y\right) ; x, y\right)\right|+T_{n, m, a_{n}}^{b_{m}, c_{m}}\left(\left|t_{1}-x\right|\right. \\
& \left.\cdot \omega^{(1)}\left(f_{x}^{\prime} ; \delta_{n}\right)\left(\frac{\left|t_{1}-x\right|}{\delta_{n}}+1\right) ; x, y\right) \\
& +T_{n, m, a_{n}}^{b_{m}, c_{m}}\left(\left|t_{2}-y\right| \omega^{(1)}\left(f_{y}^{\prime} ; \delta_{m}\right)\right. \\
& \left.\cdot\left(\frac{\left|t_{2}-y\right|}{\delta_{m}}+1\right) ; x, y\right) \text {, }
\end{aligned}
$$

Since $|\eta-x|<\left|t_{1}-x\right|$ and $|\zeta-y|<\left|t_{2}-y\right|$.

Using last inequalities, we have

$$
\begin{aligned}
\left|T_{n, m, a_{n}}^{b_{m}, c_{m}}(f ; x, y)-f(x, y)\right| \\
\leq \quad \Lambda\left|T_{n, m, a_{n}}^{b_{m}, c_{m}}\left(\left(t_{1}-x\right) ; x, y\right)\right| \\
\quad+\Upsilon\left|T_{n, m, a_{n}}^{b_{m}, c_{m}}\left(\left(t_{2}-y\right) ; x, y\right)\right| \\
\quad++\omega^{(1)}\left(f_{x}^{\prime} ; \delta_{n_{1}}\right) T_{n, m, a_{n}}^{b_{m}, c_{m}}\left(\left|t_{1}-x\right| ; x, y\right) \\
\quad+\frac{\omega^{(1)}\left(f_{x}^{\prime} ; \delta_{n_{1}}\right)}{\delta_{n_{1}}} T_{n, m, a_{n}}^{b_{m}, c_{m}}\left(\left|t_{1}-x\right|^{2} ; x, y\right) \\
\quad+\omega^{(2)}\left(f_{y}^{\prime} ; \delta_{m}\right) T_{n, m, a_{n}}^{b_{m}, c_{m}}\left(\left|t_{2}-y\right| ; x, y\right) \\
+\frac{\omega^{(2)}\left(f_{y}^{\prime} ; \delta_{n_{2}}\right)}{\delta_{m}} T_{n, m, a_{n}}^{b_{m}, c_{m}}\left(\left|t_{2}-y\right|^{2} ; x, y\right) .
\end{aligned}
$$

Now, applying the Cauchy-Schwarz inequality

$$
\begin{aligned}
& \left|T_{n, m, a_{n}}^{b_{m}, c_{m}}(f ; x, y)-f(x, y)\right| \\
& \leq \Lambda\left|T_{n, m, a_{n}}^{b_{m}, c_{m}}\left(\left(t_{1}-x\right) ; x, y\right)\right| \\
& \quad+\Upsilon\left|T_{n, m, a_{n}}^{b_{m}, c_{m}}\left(\left(t_{2}-y\right) ; x, y\right)\right|
\end{aligned}
$$

$$
\begin{aligned}
& ++\omega^{(1)}\left(f_{x}^{\prime} ; \delta_{n}\right)\left\{{ }_{x} C_{n}^{*}\left(\left(t_{1}-x\right)^{2} ; x, y\right)\right\}^{1 / 2} \\
& +\frac{\omega^{(1)}\left(f_{x}^{\prime} ; \delta_{n}\right)}{\delta_{n}}{ }_{x} C_{n}^{*}\left(\left(t_{1}-x\right)^{2} ; x, y\right) \\
& +\omega^{(2)}\left(f_{y}^{\prime} ; \delta_{m}\right)\left\{{ }_{y}^{*} S_{m}^{b_{m}, c_{m}}\left(\left(t_{2}-y\right)^{2} ; x, y\right)\right\}^{1 / 2} \\
& +\frac{\omega^{(2)}\left(f_{y}^{\prime} ; \delta_{m}\right)}{\delta_{m}}{ }_{y}^{*} S_{m}^{b_{m}, c_{m}}\left(\left(t_{2}-y\right)^{2} ; x, y\right) .
\end{aligned}
$$

Now choosing $\delta_{n}=\delta_{n}(x)$ and $\delta_{m}=\delta_{m}(y)$, we have

$$
\begin{aligned}
& \left|T_{n, m, a_{n}}^{b_{m}, c_{m}}(f ; x, y)-f(x, y)\right| \\
& \leq \Lambda \frac{a_{n}}{2 n} \\
& \quad+\Upsilon\left(\left|\frac{b_{m}^{2} \mathscr{L}^{\prime \prime}\left(b_{m} y\right)}{c_{m}^{2} \mathscr{L}^{\prime}\left(b_{m} y\right)}-1\right| y+\frac{2 \mathscr{K}^{\prime}(1)+1}{2 c_{m} \mathscr{K}(1)}\right) \\
& \quad+\omega^{(1)}\left(f_{x}^{\prime} ; \delta_{n}\right)\left(1+\sqrt{\delta_{n}}\right) \\
& \quad+\omega^{(2)}\left(f_{y}^{\prime} ; \delta_{m}\right)\left(1+\sqrt{\delta_{m}}\right) .
\end{aligned}
$$

This completes the proof.

\section{Weighted Approximation Properties}

The weighted Korovkin-type theorems are used for the purpose of this study, which are previously proved by Gadjiev $[12,13]$. Therefore we need to introduce the notations of [13]. Let $\mathbb{R}_{+}^{2}=\{(x, y): x \geq 0, y \geq 0\}$ and $B_{\rho}\left(\mathbb{R}_{+}^{2}\right)$ be the space of all functions having the property $|f(x, y)| \leq M_{f} \rho(x, y)$, where $(x, y) \in \mathbb{R}_{+}^{2}$ and $M_{f}$ is a constant depending on function only. By $C_{\rho}\left(\mathbb{R}_{+}^{2}\right)$ we denote the subspace of all continuous functions belonging to $B_{\rho}\left(\mathbb{R}_{+}^{2}\right)$. It is clear that $C_{\rho}\left(\mathbb{R}_{+}^{2}\right)$ is a linear normed space with the norm $\|f\|_{\rho}=\sup _{(x, y) \in \mathbb{R}_{+}^{2}}(|f(x, y)| / \rho(x, y))$. Also, let $C_{\rho}^{*}\left(\mathbb{R}_{+}^{2}\right)$ be the subspace of all functions $f \in C_{\rho}\left(\mathbb{R}_{+}^{2}\right)$, for which $\lim _{|(x, y)| \rightarrow \infty}\left(f(x, y) /\left(1+|(x, y)|^{2}\right)\right)=k_{f}<\infty$, where $|(x, y)|=\sqrt{x^{2}+y^{2}}$.

Theorem 10. Let $f$ belong to $C_{\rho}^{*}\left(\mathbb{R}_{+}^{2}\right)$ and $|f(x, y)| \leq$ $M_{f} \rho(x, y)$. Then

$$
\lim _{n, m \longrightarrow \infty}\left\|T_{n, m, a_{n}}^{b_{m}, c_{m}}(f ; x, y)-f\right\|_{C_{\rho}^{*}\left(R_{+}^{2}\right)}=0
$$

if and only if

(i) $\left\|T_{n, m, a_{n}}^{b_{m}, c_{m}}(1 ; x, y)-1\right\|_{C_{\rho}^{*}\left(\mathbb{R}_{+}^{2}\right)}=0$;

(ii) $\left\|T_{n, m, a_{n}}^{b_{m}, c_{m}}\left(e_{10} ; x, y\right)-x\right\|_{C_{\rho}^{*}\left(\mathbb{R}_{+}^{2}\right)}=0$;

(iii) $\left\|T_{n, m, a_{n}}^{b_{m}, c_{m}}\left(e_{01} ; x, y\right)-y\right\|_{C_{\rho}^{*}\left(\mathbb{R}_{+}^{2}\right)}=0$;

(iv) $\left\|T_{n, m, a_{n}}^{b_{m}, c_{m}}\left(e_{20} ; x, y\right)-x^{2}\right\|_{C_{\rho}^{*}\left(\mathbb{R}_{+}^{2}\right)}=0$; 
(v) $\left\|T_{n, m, a_{n}}^{b_{m}, c_{m}}\left(e_{02} ; x, y\right)-y^{2}\right\|_{C_{\rho}^{*}\left(\mathbb{R}_{+}^{2}\right)}=0 ;$

as $n, m \longrightarrow \infty$ for $(x, y) \in I_{a b}$.

Proof. The necessity part is trivial; then we need only to prove sufficiency. Let $(x, y),(s, t) \in I_{a b}$, and $f \in C_{\rho}^{*}\left(\mathbb{R}_{+}^{2}\right)$. Since for each $f \in C_{\rho}^{*}\left(\mathbb{R}_{+}^{2}\right)$ is uniformly on $I_{a b}$, for each $\epsilon>0$ there exists some $\delta>0$, such that for each $(s, t) \in I_{a b}$ with $\sqrt{(s-x)^{2}+(t-y)^{2}}<\delta$ implies $|f(s, t)-f(x, y)|<\epsilon$. Now let $(x, y) \in I_{a b}$ and $(s, t) \in \mathbb{R}_{+}^{2}$ and let $\left(x_{1}, y_{1}\right)$ be an arbitrary boundary point of $I_{a b}$ such that $0 \leq x_{1} \leq a, 0 \leq y_{1} \leq b$. Since $f$ is continuous on the boundary points also, then for each $\epsilon>0$ there exists $\delta>0$ such that $\sqrt{(s-x)^{2}+(t-y)^{2}}<\delta$ implies

$$
\begin{aligned}
|f(s, t)-f(x, y)| \leq & \left|f(s, t)-f\left(x_{1}, y_{1}\right)\right| \\
& +\left|f\left(x_{1}, y_{1}\right)-f(x, y)\right|<\epsilon .
\end{aligned}
$$

On the other hand, if $\sqrt{(s-x)^{2}+(t-y)^{2}} \geq \delta$, we have

$$
|f(s, t)-f(x, y)| \leq C\left(\frac{(s-x)^{2}+(t-y)^{2}}{\delta^{2}}\right),
$$

where $C>0$ is constant and $(x, y) \in I_{a b}$ and $(s, t) \in \mathbb{R}_{+}^{2}$. So, we get the following inequality:

$$
|f(s, t)-f(x, y)| \leq \epsilon+C\left(\frac{(s-x)^{2}+(t-y)^{2}}{\delta^{2}}\right),
$$

for $(x, y) \in I_{a b},(s, t) \in \mathbb{R}_{+}^{2}$. Now applying the operators $T_{n, m, a_{n}}^{b_{m}, c_{m}}(f ; x, y)$ in the last inequality and taking relations (i)(v) of Theorem 10, sufficiency is obtained easily.

Now we estimate the rate of approximation of the operators $T_{n, m, a_{n}}^{b_{m}, c_{m}}$ in the terms of the weighted modulus of continuity $\Omega\left(f ; \delta_{n}, \delta_{m}\right)$ (see [14]) defined by

$$
\begin{array}{r}
\Omega\left(f ; \delta_{n}, \delta_{m}\right) \\
=\sup _{(x, y) \in \mathbb{R}_{+}^{2}} \sup _{\left|h_{1}\right| \leq \delta_{1},\left|h_{2}\right| \leq \delta_{2}} \frac{\left|f\left(x+h_{1}, y+h_{2}\right)-f(x, y)\right|}{\rho(x, y) \rho(h 1, h 2)}, \\
f \in C_{\rho}^{*}\left(\mathbb{R}^{+}\right) .
\end{array}
$$

and it satisfied the following properties:

$$
\begin{aligned}
& |f(t, s)-f(x, y)| \\
& \quad \leq 8 \Omega\left(f ; \delta_{n}, \delta_{m}\right)\left(1+x^{2}+y^{2}\right) g(t, x) g(t, y)
\end{aligned}
$$

where $g(t, x)=\left(\left(1+|t-x| / \delta_{n}\right)\left(1+(t-x)^{2}\right)\right)$ and $g(s, y)=$ $\left(\left(1+|s-y| / \delta_{m}\right)\left(1+(s-y)^{2}\right)\right)$.

Theorem 11. For each $f \in C_{\rho}^{*}\left(\mathbb{R}^{+}\right)$, there exists a positive constant $B$ independent of $a_{n}, b_{m}$, and $c_{m}$ such that the inequality

$$
\left\|T_{n, m, a_{n}}^{b_{m}, c_{m}}(f ; x, y)-f\right\|_{\rho^{3}} \leq B \Omega\left(f ; \delta_{n}, \delta_{m}\right),
$$

is satisfied for a sufficiently large $n, m$, where $\delta_{n}=a_{n} / n$ and $\delta_{m}=1 / c_{m}$.

Proof. By the linearity and monotonicity of $T_{n, m, a_{n}}^{b_{m}, c_{m}}$ applied to inequality (60) we obtain

$$
\begin{aligned}
& \left|T_{n, m, a_{n}}^{b_{m}, c_{m}}(f ; x, y)(f ; x, y)-f(x, y)\right| \leq 8 \\
& \cdot \frac{n c_{m}}{a_{n} \mathscr{K}(1) \mathscr{L}\left(b_{m} y\right)} \Omega\left(f ; \delta_{n}, \delta_{m}\right)\left(1+x^{2}+y^{2}\right) \\
& \times \sum_{k=0}^{n} p_{n, k}\left(\frac{x}{a_{n}}\right) \int_{(k / n) a_{n}}^{((k+1) / n) a_{n}} g(x, t) d t \times \sum_{j=0}^{\infty} p_{j}\left(b_{m} y\right) \\
& \cdot \int_{j / c_{m}}^{(j+1) / c_{m}} g(s, y) d s \\
& \leq 8 \Omega\left(f ; \delta_{n}, \delta_{m}\right)\left(1+x^{2}+y^{2}\right)\left\{1+\frac{n}{\delta_{n} a_{n}}\right. \\
& \cdot \sum_{k=0}^{n} p_{n, k}\left(\frac{x}{a_{n}}\right) \int_{(k / n) a_{n}}^{((k+1) / n) a_{n}}|t-x| d t+\frac{n}{\delta_{n} a_{n}} \\
& \cdot \sum_{k=0}^{n} p_{n, k}\left(\frac{x}{a_{n}}\right) \int_{(k / n) a_{n}}^{((k+1) / n) a_{n}}(t-x)^{2} d t+\frac{n}{\delta_{n} a_{n}} \\
& \left.\cdot \sum_{k=0}^{n} p_{n, k}\left(\frac{x}{a_{n}}\right) \int_{(k / n) a_{n}}^{((k+1) / n) a_{n}}|t-x|(t-x)^{2} d t\right\} \\
& \times\left\{1+\frac{c_{m}}{\delta_{m} \mathscr{K}(1) \mathscr{L}\left(b_{m} y\right)} \sum_{j=0}^{\infty} p_{j}\left(b_{m} y\right)\right. \\
& \cdot \int_{j / c_{m}}^{(j+1) / c_{m}}|s-y| d s+\frac{c_{m}}{\delta_{m} \mathscr{K}(1) \mathscr{L}\left(b_{m} y\right)} \\
& \cdot \sum_{j=0}^{\infty} p_{j}\left(b_{m} y\right) \int_{j / c_{m}}^{(j+1) / c_{m}}|s-y|(s-y)^{2} d s \\
& +\frac{c_{m}}{\mathscr{K}(1) \mathscr{L}\left(b_{m} y\right)} \sum_{j=0}^{\infty} p_{j}\left(b_{m} y\right) \\
& \left.\cdot \int_{j / c_{m}}^{(j+1) / c_{m}}(s-y)^{2} d s\right\}
\end{aligned}
$$

where $g(x, t)=\left(\left(1+|t-x| / \delta_{n}\right)\left(1+(t-x)^{2}\right)\right)$ and $g(s, y)=$ $\left(\left(1+|s-y| / \delta_{m}\right)\left(1+(s-y)^{2}\right)\right)$.

Applying Cauchy-Schwarz inequality, we have

$$
\begin{aligned}
& \left|T_{n, m, a_{n}}^{b_{m}, c_{m}}(f ; x, y)-f(x, y)\right| \\
& \quad \leq 8 \Omega\left(f ; \delta_{n}, \delta_{m}\right)\left(1+x^{2}+y^{2}\right)
\end{aligned}
$$




$$
\begin{aligned}
& \times\left\{1+\frac{\sqrt{\Delta_{1}}}{\delta_{n}}+\delta_{1}+\frac{\sqrt{\Delta_{1} \Delta_{2}}}{\delta_{n}}\right\} \\
& \times\left\{1+\frac{\sqrt{\Delta_{1}^{*}}}{\delta_{n}}+\delta_{1}+\frac{\sqrt{\Delta_{1}^{*} \Delta_{2}^{*}}}{\delta_{n}}\right\} .
\end{aligned}
$$

where $\Delta_{1}, \Delta_{1}^{*} \Delta_{2}$, and $\Delta_{2}^{*}$ are defined by Lemma 2 .

Combining (64) and all identities in Lemma 2, we obtain

$$
\begin{aligned}
& \left|T_{n, m, a_{n}}^{b_{m}, c_{m}}(f ; x, y)-f(x, y)\right| \leq 8 \Omega\left(f ; \delta_{n}, \delta_{m}\right)\left(1+x^{2}\right. \\
& \left.+y^{2}\right) \times\left\{1+\frac{1}{\delta_{n}} \sqrt{O\left(\frac{a_{n}}{n}\right) \sum_{i=1}^{2} x^{i}}\right. \\
& \left.+O\left(\frac{a_{n}}{n}\right) \sum_{i=1}^{2} x^{i} \frac{1}{\delta_{n}} \sqrt{O\left(\frac{a_{n}}{n}\right) \sum_{i=1}^{2} x^{i} O\left(\frac{a_{n}}{n}\right) \sum_{i=1}^{4} x^{i}}\right\} \\
& \quad \times\left\{1+\frac{1}{\delta_{m}} \sqrt{O\left(\frac{1}{c_{m}}\right)\left(\sum_{i=1}^{2} y^{i}\right)}\right. \\
& +O\left(\frac{1}{c_{m}}\right)\left(\sum_{i=1}^{2} y^{i}\right) \\
& \left.+\frac{1}{\delta_{m}} \sqrt{O\left(\frac{1}{c_{m}}\right)\left(\sum_{i=1}^{2} y^{i}\right) O\left(\frac{1}{c_{m}}\right)\left(\sum_{i=1}^{4} y^{i}\right)}\right\}
\end{aligned}
$$

Choosing $\delta_{n}=a_{n} / n$ and $\delta_{m}=1 / c_{m}$, and for sufficiently large value of $n$ and $m$, we obtain

$$
\left\|T_{n, m, a_{n}}^{b_{m}, c_{m}}(f ; x, y)-f\right\|_{\rho^{3}} \leq B \Omega\left(f ; \delta_{n}, \delta_{m}\right)
$$

where $\mathrm{B}$ is a constant independent of $a_{n}, b_{m}$, and $c_{m}$.

\section{Conclusion}

We studied a new sequence generalization of the SzaszKantorovich-Chlodowsky type operators defined by means of the Brenke type polynomials defined by (9). This type of modification enables better error estimation for a certain function in comparison to the Szász-Kantorovich-Chlodowsky operators and Szasz-Chlodowsky-type operators. We find the rate of convergence using weighted Korovkin-type theorem. We give some inequalities for these operators means of partial and full modulus of continuity and also obtain a Lipschitz type theorem. At the end, we mentioned results on the weighted modulus of continuity due to Ispir for the operators $T_{n, m, a_{n}}^{b_{m}, c_{m}}$.

\section{Data Availability}

The data used to support the findings of this study are included within the article.

\section{Conflicts of Interest}

The authors declare that they have no conflicts of interest.

\section{References}

[1] J. Favard, "Sur les multiplicateurs d'interpolation," Journal de Mathématiques Pures et Appliquées, vol. 9, no. 23, 1944.

[2] A. Jakimovski and D. Leviatan, "Generalized Szász operators for the approximation in the infinite interval," Mathematica-Revue d'Analyse Numérique et de Théorie de l'Approximation, vol. 11, no. 34, pp. 97-103, 1969.

[3] S. Varma, S. Sucu, and G. Içoz, "Generalization of Szasz operators involving Brenke type polynomials," Computers and Mathematics with Applications, vol. 64, no. 2, pp. 121-127, 2012.

[4] A. Çigdem and I. Buyukyazici, "Approximation by kantorovichszasz type operators based on brenke type polynomials," Numerical Functional Analysis and Optimization, vol. 37, no. 12, pp. 1488-1502, 2016

[5] B. Baxhaku and P. N. Agrawal, "Degree of approximation for bivariate extension of Chlodowsky-type q-Bernstein-StancuKantorovich operators," Applied Mathematics and Computation, vol. 306, pp. 56-72, 2017.

[6] I. Büyükuazici and H. Sharma, "Approximation properties of two-dimensional q-Bernstein-Chlodowsky-Durrmeyer operators," Numerical Functional Analysis and Optimization, vol. 33, no. 12, pp. 1351-1371, 2012.

[7] M. Sidharth, A. M. Acu, and P. N. Agrawal, "Chlodowsky-SzaszAppell-type operators for functions of two variables," Annals of Functional Analysis, vol. 8, no. 4, pp. 446-459, 2017.

[8] P. N. Agrawal and N. Ispir, "Degree of approximation for bivariate Chlodowsky-Szasz-CHArlier type operators," Results in Mathematics, vol. 69, no. 3-4, pp. 369-385, 2016.

[9] N. Ispir and I. Büyükuazici, "Quantitative estimates for a certain bivariate Chlodowsky-Szasz-Kantorovich type operators," Mathematical Communications, vol. 21, no. 1, pp. 31-44, 2016.

[10] P. N. Agrawal, B. Baxhaku, and R. Chauhan, "The approximation of bivariate Chlodowsky-Szajsz-Kantorovich-Charliertype operators," Journal of Inequalities and Applications, vol. 1, p. 195, 2017.

[11] V. I. Volkov, "On the convergence of sequences of linear positive operators in the space of continuous functions of two variables," Doklady Akademii Nauk SSSR, vol. 115, pp. 17-19, 1957.

[12] A. Gadjev, "Positive linear operators in weighted spaces of functions of several variables," izv. akad. nauk Azerbaijan ssr ser. fiz. Tehn. Mat. Nauk, vol. 1, no. 4, pp. 32-37, 1980.

[13] A. Gadjev and H. Hacisalihoglu, Convergence of the Sequences of Linear Positive Operators, Ankara University, 1995.

[14] N. Ispir and A. Çigdem, "Approximation by modified SzaszMirakjan operators on weighted spaces," The Proceedings of the Indian Academy of Sciences - Mathematical Sciences, vol. 112, no. 4, pp. 571-578, 2002. 


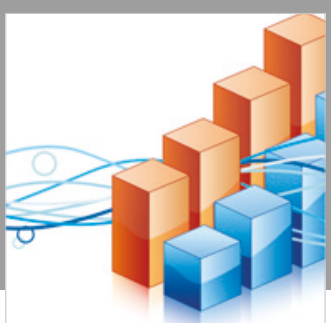

Advances in

Operations Research

\section{-n-m}
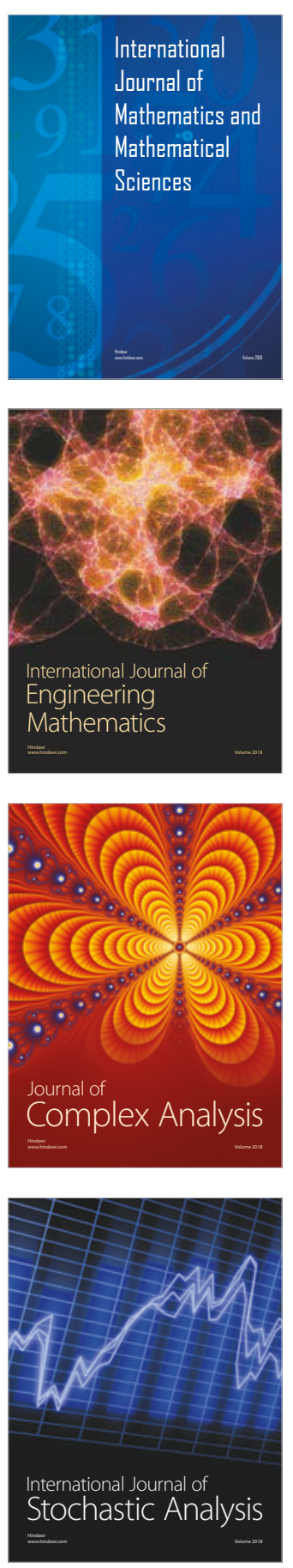
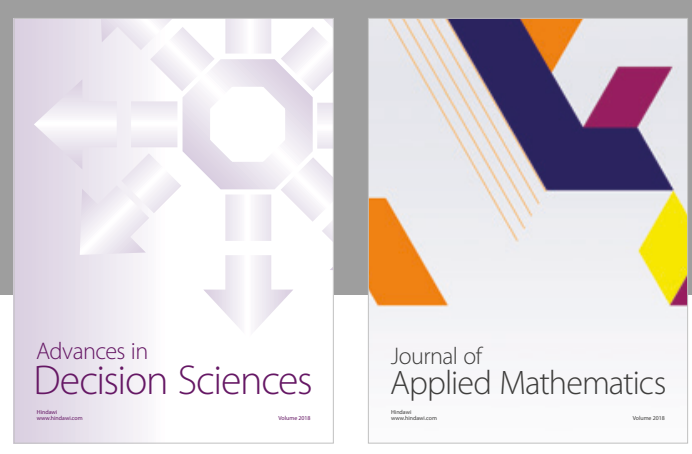

Journal of

Applied Mathematics
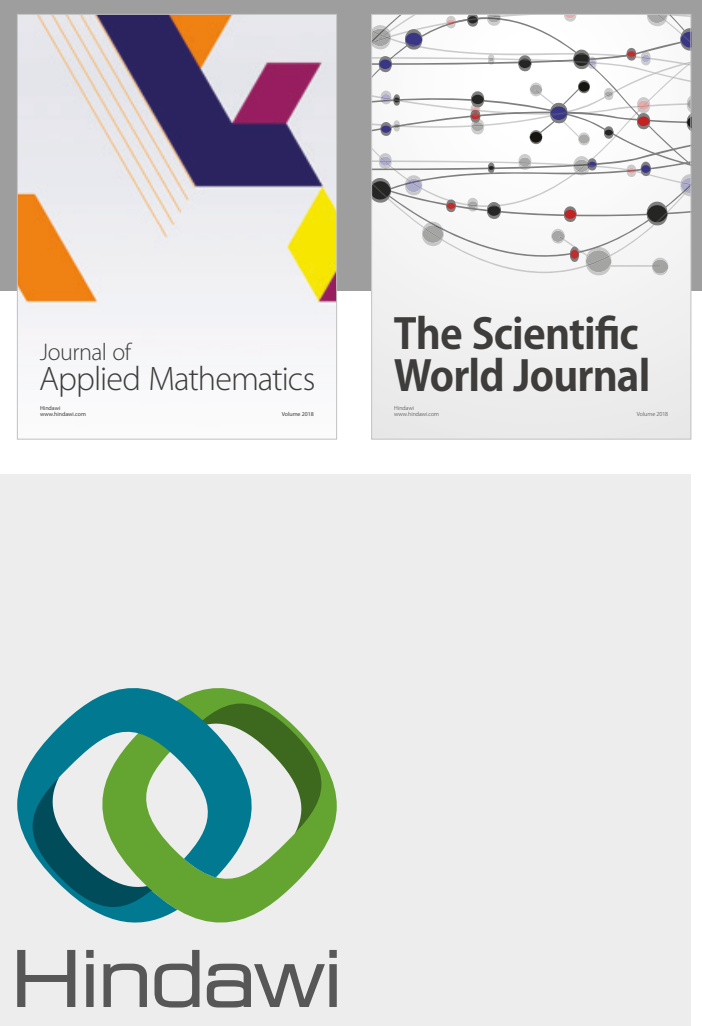

Submit your manuscripts at

www.hindawi.com

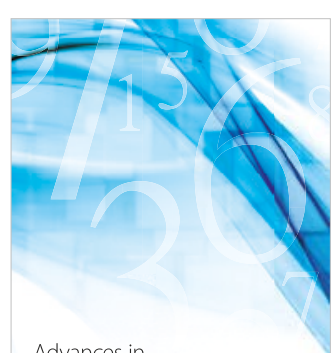

Advances in
Numerical Analysis
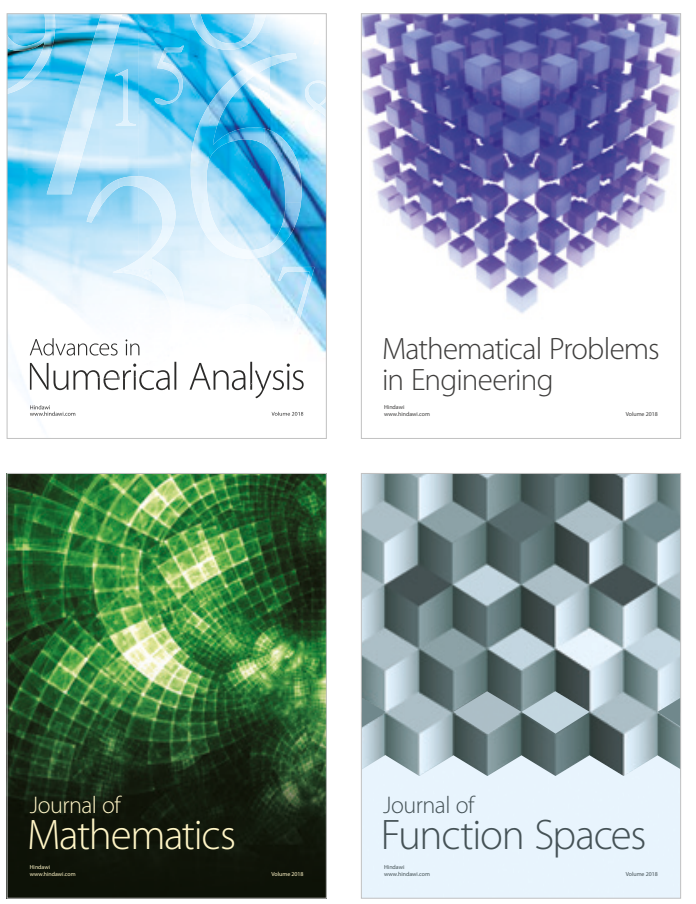

Mathematical Problems in Engineering

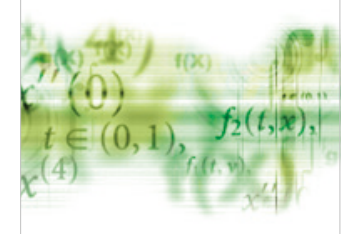

International Journal of

Differential Equations

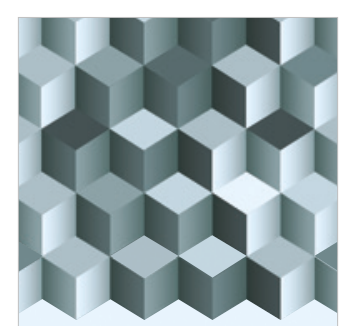

Journal of

Function Spaces

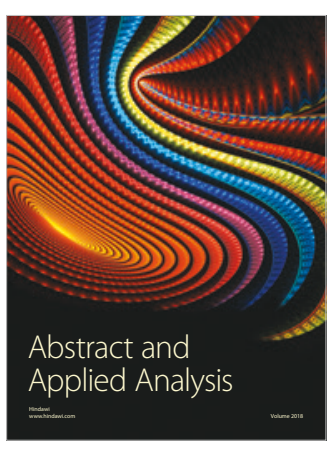

The Scientific

World Journal

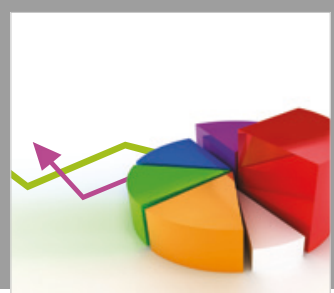

Journal of

Probability and Statistics
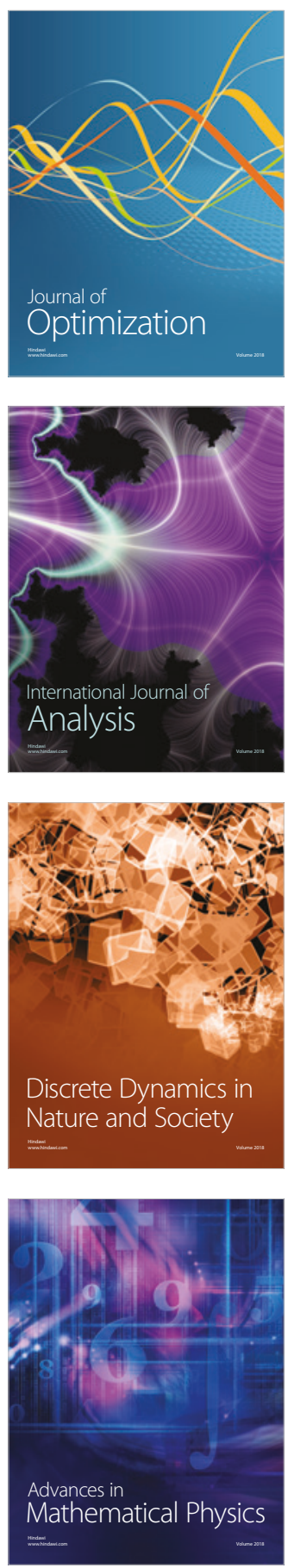